\title{
A importância da busca ativa do enfermeiro na atenção primária para prevenção do câncer de colo uterino
}

The importance of active nurse search in primary care for prevention of uterine cancer

La importância del búsqueda activa de infermero em la atención primária para la prevención del cáncer de cuello uterino

Gabriela de Carvalho Braga Nazaré ${ }^{1}$, Jussara Camila Ribeiro ${ }^{1 *}$, Andréia Andrade dos Santos ${ }^{1}$, Jane

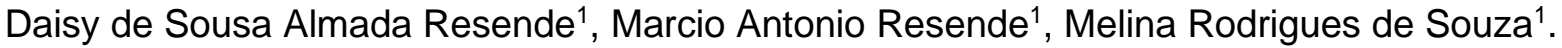

\section{RESUMO}

Objetivo: Identificar a atuação do enfermeiro da Equipe Saúde da Família na prevenção do câncer de colo uterino e propor estratégias para minimizar estes índices. Métodos: Trata-se de uma pesquisa de revisão de literatura, que foi realizada por meio de verificação sistemática de artigos indexados e publicados em base de dados da Biblioteca Virtual da Saúde (BVS). Foi utilizado com metodologia, pesquisa de revisão de literatura, por meio de verificação sistemática de artigos indexados e publicados em sites oficiais. Resultado: Ressalta-se que o enfermeiro tem um papel importante na detecção precoce deste câncer e nas ações de rastreamento, tendo em vista ser atribuição de este profissional realizar assistência integral a mulher. Conclusão: Nesse sentido, no cenário da prevenção do câncer de colo do útero, o exame de Papanicolau, é o método de escolha na detecção precoce do carcinoma cervical, sendo Enfermeiro responsável por proporcionar atendimento integral, esclarecimento e rastreamento adequado no diagnóstico, tratamento, acompanhamento e consequente redução de danos.

Palavras-Chave: Teste de Papanicolau, Relações enfermeiro-paciente, Neoplasias do colo do útero, Prevenção primária.

\begin{abstract}
ABSTRAT
Objective: To identify the role of the nurse of the Family Health Team in the prevention of cervical cancer and propose strategies to minimize these rates. Methods: This is a literature review survey, which was carried out through systematic verification of indexed articles published in the Virtual Health Library (VHL) database. It was used with methodology, literature review research, through systematic verification of indexed articles published on official websites. Results: It is noteworthy that nurses play an important role in the early detection of this cancer and in screening actions, in order to be attributed to this professional to provide comprehensive care to women. Conclusion: In this sense, in the scenario of cervical cancer prevention, the pap smear is the method of choice in the early detection of cervical carcinoma, being a nurse responsible for providing comprehensive care, clarification and appropriate screening in diagnosis, treatment, monitoring and consequent harm reduction.
\end{abstract}

Keywords: Pap smear, Nurse-patient relations, Cervical neoplasms, Primary prevention.

\footnotetext{
${ }^{1}$ Centro Universitário Presidente Tancredo de Almeida Neves (UNIPTAN). São João Del Rei, MG.

*E-mail: jussaralucas2015@hotmail.com
}

SUBMETIDO EM: 10/2019

ACEITO EM: 11/2019

PUBLICADO EM: 1/2020 


\section{RESUMEN}

Objetivo: Identificar el papel de la enfermera del Equipo de Salud Familiar en la prevención del cáncer de cuello uterino y proponer estrategias para minimizar estas tasas. Método: Se trata de una encuesta de revisión de la literatura, que se realizó mediante la verificación sistemática de los artículos indexados publicados en la base de datos de la Biblioteca Virtual en Salud (BVS). Se utilizó con la metodología, la investigación de revisión de la literatura, a través de la verificación sistemática de los artículos indexados publicados en los sitios web oficiales. Resultados: Cabe destacar que las enfermeras juegan un papel importante en la detección precoz de este cáncer y en las acciones de cribado, con el fin de atribuir a este profesional la atención integral a las mujeres. Conclusión: En este sentido, en el escenario de la prevención del cáncer de cuello uterino, el frotis de Papanicolaou es el método de elección en la detección precoz del carcinoma de cuello uterino, siendo una enfermera responsable de proporcionar atención integral, aclaración y cribado adecuado en el diagnóstico, tratamiento, seguimiento y consecuente reducción de daños.

Palabras clave: Papanicolaou, Relaciones enfermera-paciente, Neoplasias cervicales, Prevención primaria.

\section{INTRODUÇÃO}

O câncer de colo de útero (CCU) é um tumor maligno provocado pelo desenvolvimento acelerado e desorientado das células que envolvem o tecido epitelial do útero (BRASIL, 2017). Uma das causas mais frequentes do CCU é o contágio permanente do Papiloma Vírus Humano (HPV), através do ato sexual sem preservativo (NAKAGAWA JTT, SCHIRMER J, BARBIERI M, 2010).

Com aproximadamente 530 mil casos novos por ano no mundo, o CCU está no ranking dos três mais comuns entre as mulheres, sendo responsável pelo óbito de 274 mil por ano (WHO, 2008; WHO, 2014). Atualmente, o Brasil, é considerado o terceiro país em maior incidência de neoplasia nas mulheres, entre eles, o CCU é o grande vilão na saúde pública em decorrência do alto índice de incidência e mortalidade (BRASIL, 2010).

Frigato S (2003) relata que dentre as causas que estão elencadas ao desenvolvimento do câncer do colo do útero, o papiloma vírus humano (HPV) é considerado como a principal, porém existem outros fatores relacionados aos hábitos de vida como tabagismo, fatores socioeconômicos, o início precoce da vida sexual, a multiplicidade de parceiros, fatores ambientais e hábitos impróprios de higiene íntima.

Logo, para prevenção e detecção precoce do câncer do colo do útero é fundamental que seja realizado periodicamente o exame citopatológico oncótico, sendo esse um método eficaz para identificação prévia de possíveis alterações citológicas que podem levar ao desenvolvimento do câncer (BRASIL, 2010; BRASIL, 2011).

Para realização de tal exame, a principal porta de entrada do paciente no sistema de saúde deve ser na Atenção Básica $(\mathrm{AB})$ de forma descentralizada, próxima ao usuário, sendo, portanto, local oportuno para a realização de atividades educativas quanto ao controle do câncer do colo do útero. $A A B$ tem, entre suas atribuições, coordenar o cuidado e ordenar as redes de atenção à saúde. Assim, ela acompanha os usuários longitudinalmente além de desenvolver ações de promoção à saúde, prevenção e detecção precoce, tratamentos e reabilitações (BRASIL, 2010; BRASIL, 2011).

Além disto, de acordo com Brasil (2011), os profissionais que trabalham na Equipe Saúde da Família (ESF) possuem uma área adscrita, o que possibilita o conhecimento da sua comunidade e a busca ativa dessas usuárias para a realização da citologia com técnica padronizada no intuito de obter diagnóstico precoce e tratamento apropriado dos casos com alterações.

Ressalta-se que o enfermeiro tem um papel importante na detecção precoce do câncer do colo do útero e nas ações de rastreamento, tendo em vista ser atribuição do enfermeiro realizar atenção total a paciente, por meio de atendimento e exame de Papanicolau (BRASIL 2010). 
É ele que obtém o conhecimento, peça fundamental na busca por uma prevenção de qualidade, assim como os agentes de saúde e os profissionais médicos que buscam alcançar e tornar eficientes os programas instituídos pelo Ministério da Saúde, tornando a Atenção Básica o centro dos esforços para melhorar a saúde e o bem-estar da população PINTO JEP, AQUINO R, MEDINA MG, et.al., 2018).

Diante desse cenário que envolve a prevenção do $\mathrm{CCU}$, tem-se a problemática deste estudo, que consiste no aumento exacerbado dos índices de mulheres com câncer de colo de útero em todas as faixas etárias, que poderiam ser reduzido se a este nível de atenção à saúde fosse priorizado os desafios emergentes que ameaçam a saúde e o bem-estar no futuro. Cabe-se também refletir no que se refere ao investimento de custeio, tendo em vista que há evidências de que a atenção primária de qualidade reduz os gastos totais em saúde e melhora a eficiência, como por exemplo, minimizando as internações hospitalares. Desse modo, internações que poderiam ser evitadas com detecção precoce de enfermidades, oneram o orçamento público da saúde e desperdiçam os recursos que poderiam ser utilizados para o custeio de outras ações em saúde (PINTO JEP, AQUINO R, MEDINA MG, et.al., 2018).

Justifica-se a importância desta pesquisa, no intuito de destacar a necessidade do enfermeiro em traçar melhores estratégias para a prevenção do CCU e assim, reduzir os índices de morbimortalidade da doença. Portanto, a pesquisa tem o objetivo de identificar a atuação do enfermeiro da Equipe Saúde da Família na prevenção do câncer de colo uterino e propor estratégias para minimizar estes índices.

\section{DISCUSSÂO}

\section{Contextualizando o câncer de colo uterino}

Considerado um dos grandes vilões no Brasil e no mundo, o câncer do colo uterino representa 10\% de câncer em mulheres, apresentando 265 mil mortes por ano O CCU é incomum em mulheres até 30 anos, sendo seu maior predomínio entre 45 e 50 anos (BRASIL, 2014).

O câncer pode ser classificado como carcinoma não invasivo, que é a formação inicial em que o CCU se apresenta. Na fase inicial as partículas modificadas estão apenas no colo do útero, com alto índice de cura com tratamento precoce. Outra classificação seria o câncer invasivo, as células espalham-se para a corrente sanguínea, podendo expandir para outras estruturas do corpo, definido como metástase (BRASIL, 2014).

É possível ordenar as lesões neoplásicas intraepiteliais cervicais (NIC), a partir do tamanho de epitélio acometido por células cancerígenas, em: NIC I, de grau inferior e anomalias leves, fase pré invasiva, NIC II e III, anomalias de média e alta intensidade, que exigem supervisão e tratamento por conter quantidade significativa de células diferenciadas (PEREIRA GG, SILVA JGF, BIZELLI DFP, et al., 2018).

A enfermidade não possui etiologia específica, sendo de maior prevalência as causas externas como, práticas e modos de vida inadequados, uso prolongado de hormônios orais, vida sexual precoce, tabagismo, imunossupressão, múltiplos parceiros, prática sexual sem uso de preservativo, alteração genética e HPV (COSTA FKM, WEIGERT SP, BURCI L, et. al, 2017).

Ressalta-se que em mulheres abaixo de 25 anos, a causa mais frequente do CCU é o contágio permanente do HPV que acomete principalmente quem praticam sexo sem preservativo (FREITAS HG, SILVA MA, THULER LCS, 2012). Os sinais e sintomas se dá pela ocorrência lenta, assintomática em sua fase pré-clínica, podendo desencadear metrorragia eventualmente ou após ato sexual, leucorréia e desconforto pélvico. Em fase avançada a mulher apresenta náuseas, emagrecimento e fraqueza (BRASIL, 2014).

É uma doença com alto potencial para prevenção e cura, sendo a detecção precoce o método que proporciona o melhor tratamento, se diagnosticado na fase pré-clínica, o que atinge chance de $100 \%$ de recuperação, ressaltando a importância da consulta de enfermagem na busca do atendimento integral. Além disto, ainda que 
a sociedade tenha acesso a várias informações disponíveis de forma gratuita, cabe ressaltar a necessidade de "educar" a população, bem como de orientar sobre a disponibilidade do exame pela rede pública (BRASIL, 2011).

Entre os principais tipos de tratamento estão a histerectomia do colo de útero, a radioterapia e a quimioterapia. O tratamento de escolha depende do estágio clínico da doença, extensão do tumor e história pessoal e social (BRASIL, 2016).

A histerectomia compreende a retirada parcial (remoção da parte superior do colo do útero) ou total do colo uterino, sendo o primeiro tipo de tratamento escolhido para o CCU e o segundo procedimento cirúrgico mais utilizado no Brasil (CESAR MAP, ANTUNES LB, AGUIAR RM, 2010).

No tratamento por radioterapia, são utilizados raios ionizantes, a fim de destruir ou inibir o desenvolvimento de células do câncer. A quimioterapia é realizada através de medicamentos endovenosos ou orais que obtém a circulação sistêmica e atinge todos os órgãos, tratando assim também as metástases. É empregado em ciclos de dias, com pausa para o organismo se reabilitar. Dependendo da fase do CCU, o tratamento pode ser combinado entre radioterapia e quimioterapia, sendo a segunda potencializante da primeira. (BRASIL, 2017).

\section{As políticas públicas de atenção à saúde da mulher}

Surge na década de 80 um programa de atenção à saúde da mulher, mas suas diretrizes foram validadas e expandidas após 20 anos. Este programa é focado nos princípios da humanização e um bom atendimento, buscando resolver questões da saúde feminina, identificando e buscando seus direitos. Tal programa é baseado na descentralização, hierarquização e regionalização dos serviços, propondo ações educativas para melhor conhecimento do corpo feminino (LEMOS A, 2011).

Logo, tornou-se maior o olhar das ações de integralidade sobre elas, passando a ter um panorama abrangente de saúde. Com a criação do programa as mulheres não são mais consideradas somente produtoras e reprodutoras, como era antes, tendo a mesma o poder para decidir seus critérios reprodutivos. O programa de atenção integral a saúde da mulher (PAISM) apresenta fases, período de vida, condições da saúde feminina e concepção, adquirindo maior eficácia após a chegada do Sistema Único de Saúde (SUS) com a Constituição Federal de 1988 (FERREIRA MH, SALES MC, 2017).

Uma das políticas do governo de atenção integral e prevenção à saúde da mulher foi criado em 1984 o programa Viva a Mulher, fazendo uma busca ativa das pacientes que a muito tempo não realizavam o exame Papanicolau. Com o objetivo de diminuir a letalidade e alterações físicas, psicológicas e comunitárias desses carcinomas em mulheres, oferecendo atividades para precaução e diagnósticos em fase precoce, terapêutica e reparação (BRASIL, 2018). Assim, a partir do ano de 2000 as ações foram intensificadas e aumentou significativamente o número de exames realizados na atenção básica para detecção precoce do CCU. Em 2014 através do Programa Nacional de Imunizações (PNI), foi instituída a imunização de meninas entre 11 e 13 anos ao combate do vírus HPV (BRASIL, 2014).

Tal vacina é uma forma segura e eficiente para prevenção, combate e difusão à patologia. No Brasil, introduziuse duas formas de imunização profilática, a bivalente e a quadrivalente, ambas possuem a proteína $L 1$, são desenvolvidas através de tecnologia combinante propositando ter fração viral análoga de gêneros mais comuns do CCU, que são o HPV16 e o HPV18 (bivalente), presentes em 70\% dos casos da patologia e a imunização quadrivalente previne ainda os tipos 6 e 11 de HPV, ambas são consideradas de alta eficácia, estimativas apontam que se toda a população alvo for imunizada as taxas de câncer cervical serão reduzidas em dois terços. (ZARDO GP, FARAH FP, MENDES FG, et.al., 2014)

Contudo, a estratégia de rastreamento no Brasil, de acordo com o Ministério da Saúde, é o exame citopatológico focado em mulheres de 25 a 64 anos, principalmente as mulheres sexualmente ativas, para identificar casos confirmados, diagnosticar e aplicar o melhor tratamento. Portanto, faz-se necessário garantir a 
integralidade, organização e a qualidade dos programas de rastreamento, assim como o seguimento das pacientes ao programa (BRASIL, 2014).

Vale ressaltar que a incidência do carcinoma é menor em mulheres até 24 anos e a maior parte dos eventos identificados no estágio I. Assim, explica-se a recomendação de idade preconizada para início da realização do exame Papanicolau (BRASIL, 2011; SILVA, 2014).

O intervalo entre a análise deve ser de 3 anos, posteriormente a 2 análises negativas, realizada em anos consecutivos. A análise deve ser feita até os 64 anos e cessada com dois resultados negativos sucessivos nos últimos cinco anos, mulheres acima dessa idade e que ainda não fizeram o exame indica-se a realização da análise de 1 a 3 anos. Se todos apresentarem resultado negativo, elas podem abster-se da coleta (BRASIL, 2011).

\section{O papel da enfermagem no cuidado precoce do câncer de colo uterino}

Surge como ferramenta indispensável do cuidar, o papel do profissional de Enfermagem no setor primário de atenção à saúde, o qual atua principalmente com promoção e prevenção das patologias, incluindo prevenção de câncer de colo de útero. Com foco de cuidado na saúde feminina, o enfermeiro deve traçar maneiras de busca ativa da população para realização do exame de Papanicolau (SILVEIRA L, MAIA RCB, CARVALHO MF, et.al., 2018).

Tal exame é desenvolvido também por meio de atividades da Estratégia e Saúde da Família (ESF), programa este, que visa à reorganização da assistência em saúde, trabalha a família de forma integral, e não somente o indivíduo na fase da doença, trabalhando na forma de prevenção sobre essa população. Este por sua vez é uma importante ferramenta do cuidar e simultaneamente do prevenir como centro da atenção, e não mais o doente (SILVEIRA L, MAIA RCB, CARVALHO MF, et.al.,2018)

As atividades desenvolvidas do Enfermeiro na Equipe Saúde da Família (ESF) são realizadas de várias maneiras: atendimento do enfermeiro, coleta de Papanicolau, ações didáticas com a equipe do setor e população de abrangência, coordenação de recursos disponíveis, coleta eficiente de material, comunicação entre os membros da equipe e paciente e encaminhamento para os devidos fins necessários (MELO MCCSC, VILELA F, SALIMENA AMO et al., 2012).

O exame preventivo, ofertado pelo SUS, reconhece modificações no colo uterino e é a principal análise para detecção precoce do CCU. Para um diagnóstico preciso, o enfermeiro deve orientar a paciente que ela deve abster-se de ato sexual, não fazer uso de ducha, cremes íntimos dois dias anteriores a coleta. A mulher não deve estar em período menstrual, pois altera o resultado do esfregaço. Antes da coleta deve ser realizada uma anamnese da história ginecológica (COSTA FKM, WEIGERT SP, BURCI L, et.al., 2017).

No exame, a mulher deve ficar na posição litotômia, é inserido um espéculo na vagina, fazendo análise tanta da mesma como também da cérvice. Logo após, o enfermeiro realiza a coleta de material celular da endocérvice e ectocérvice, utilizando espátula e escova cervical. O material colhido é depositado em uma placa de vidro, embebido em frasco com álcool 70\% e encaminhado para estudos citopatológicos (COSTA FKM, WEIGERT SP, BURCI L, et. al., 2017).

Não é possível avaliar a amostra quando apresentar células abaixo de $10 \%$ de matéria orgânica na lâmina e análise dificultada devido a presença de contaminantes como sangue em mais de $75 \%$ do material. Para uma avaliação fidedigna deve-se apresentar boa concentração de células, espalhadas, afixadas, rosadas. (BRASIL, 2011). Assim, é função da enfermagem prestar atendimento integral as pacientes e avaliação ginecológica. Com tudo, ainda que seja de fácil detecção e cura na fase inicial, o diagnóstico do tumor uterino quase sempre é realizado de forma tardia, aumentando a taxa morbimortalidade. (CUNHA, 2015). 


\section{Atividades de educação em saúde na prevenção do CCU}

O controle do CCU está sujeito a ações voltadas para a saúde, prevenção do câncer e qualidade de vida, assim o enfermeiro intervém nessas ações e outras como as visitas domiciliares, motivando as mulheres para a realização do exame Papanicolau uma vez ao ano e a consulta de enfermagem de forma humanizada e integralizada (ZARDO GP, FARAH FP, MENDES FG, et. al., 2014).

Para melhorar essa busca ativa, deve-se adotar um formulário para registro das mulheres da microárea da ESF de cada bairro, para realização do Papanicolau e verificar a regularidade com que as mesmas fazem o exame e detectar o intervalo de idades que apresentam maior e menor adesão (COSTA FKM., WEIGERT SP, BURC L, et.al., 2017).

Uma alternativa para aumentar a adesão ao Papanicolau seria a coleta da amostra uma vez ao mês no sábado ou pelo menos duas vezes ao mês após as 18 horas, assim mulheres que não podem comparecer durante o horário de trabalho ou por outros motivos, teriam uma segunda opção para coleta. Realizar agendamento de coleta de forma simples, e rápida, de modo que não haja fila e superlotação, contar com o envolvimento dos profissionais da unidade para prestar um atendimento de qualidade (SILVEIRA L, MAIA RCB, CARVALHO MF, et.al., 2018).

Outra estratégia seria reuniões mensais com a equipe de saúde demonstrando o atual número de coletas de Papanicolau em comparação com o que é instituído pelo Ministério da Saúde, motivando a equipe para atingir o que é preconizado, sendo a equipe recompensada quando atingir ou aproximar-se da meta (MISTURA C, SILVA RCCS, SALES JRP, et. al., 2011). Nas unidades de saúde que contam com acadêmicos, é importante conversar previamente com a mulher sobre a possibilidade de participação dos mesmos durante o exame, caso ela aceite, é necessário dosar o número desses estudantes para evitar constrangimento e que ela retorne nos próximos anos (MELO MCSC, VILELA F, SALIMENA AMO, et al., 2012).

De suma importância no trabalho da equipe de enfermagem vem de encontro a conscientização aos pais sobre a vacinação contra o HPV em meninas entre 9 a 14 anos de idade. Segundo a Organização da Saúde (OMS), uma a cada 10 mulheres contraem o vírus, sendo descobertos 500 mil novos episódios de câncer cervical por ano, cerca de $70 \%$ estão registrados em países subdesenvolvidos e em desenvolvimento, destas aproximadamente 231 mil pacientes irão evoluir a óbito pelo CCU (ZARDO GP., FARAH FP, MENDES FG, et. al., 2014). É fundamental educar a sociedade sobre uso do preservativo devido a sua importância na precaução de doenças sexualmente transmissíveis, principalmente o HPV. O enfermeiro deve orientar também, uma alimentação adequada, evitar o uso do tabaco e capacitar os profissionais para a busca ativa (MISTURA C, SILVA RCCS, SALES JRP, et. al., 2011).

Além disto, o profissional de enfermagem deve desenvolver ações didáticas com práticas de atividades físicas e música para interação profissional e paciente, deixando a mesma à vontade para expor suas dúvidas. Podendo utilizar imagens, modelo anatômico reprodutor feminino, para melhor demonstração (MISTURA C, SILVA RCCS, SALES JRP, et. al., 2011).

A fim de uma melhor aceitação é necessário a realização de palestras interativas em escolas, distribuição de panfletos, grupos educativos nos ESF de cada microárea abordando como assunto o CCU e a importância da prevenção e detecção precoce, buscando apoio de outros profissionais capacitados sobre o assunto (COSTA FKM, WEIGERT SP, BURCI L, et. al., 2017).

\section{CONSIDERAÇÕES FINAIS}

Esse artigo mostra a importância da busca ativa com ações didáticas, detecção precoce e trabalho do enfermeiro na atenção básica visando prevenção e promoção do CCU na saúde da mulher de acordo com as 
políticas públicas. Salientando que apenas a procura por livre demanda das mulheres não é suficiente para cobertura eficaz do exame. As políticas públicas representam um esforço no sentido de melhorar o rastreamento do câncer proporcionando um atendimento humanizado e integral. O enfermeiro é, portanto, importante no processo de elaboração de estratégias para saúde pública, de qualidade e universal, e pode inclusive contribuir para a efetivação de políticas à saúde da mulher, ele deve sempre estar atualizado para orientar as pacientes no tocante ao câncer de colo de útero, moléstia de grande gravidade, de alta incidência no Brasil e no mundo.

\section{REFERÊNCIAS}

1. BRASIL, Instituto Oncoguia, 2017 disponível em http://www.oncoguia.org.br/conteudo/radioterapia-para-cancer-de-colodo-utero/1288/285/, acesso em 22 de junho de 2019.

2. BRASIL, Ministério da Saúde. Secretaria de Vigilância em Saúde. Secretaria de Atenção à Saúde. Política Nacional de Promoção da Saúde. 3. ed. Brasília, 2010.BRASIL. Instituto Nacional de Câncer José Alencar Gomes da Silva. ABC do câncer: abordagens básicas para o controle do câncer / Instituto Nacional de Câncer José Alencar Gomes da Silva; Organização Mario Jorge Sobreira da Silva. - 3. ed. rev. atual. - Rio de Janeiro: Inca, 2017. 108p

3. BRASIL. Instituto Nacional de Câncer José Alencar Gomes da Silva. Coordenação de Prevenção e Vigilância Estimativa 2014: Incidência de Câncer no Brasil / Instituto Nacional de Câncer José Alencar Gomes da Silva, Coordenação de Prevenção e Vigilância. Rio de Janeiro: INCA, 2014. 124p.

4. BRASIL. Instituto Nacional de Câncer José Alencar Gomes da Silva. Coordenação de Prevenção e Vigilância. Divisão de Detecção Precoce e Apoio à Organização de Rede. Diretrizes brasileiras para o rastreamento do câncer do colo do útero / Instituto Nacional de Câncer José Alencar Gomes da Silva. Coordenação de Prevenção e Vigilância. Divisão de Detecção Precoce e Apoio à Organização de Rede. - 2. ed. rev. atual. - Rio de Janeiro: INCA, 2016.

5. BRASIL. Instituto Nacional de Câncer José Alencar Gomes da Silva. Viva Mulher 20 anos: história e memória do controle do câncer do colo do útero e de mama no Brasil: catálogo de documentos / Instituto Nacional de Câncer José Alencar Gomes da Silva. - Rio de Janeiro: Inca, 2018. 86p.

6. BRASIL. Instituto Nacional de Câncer. Coordenação Geral de Ações Estratégicas. Divisão de Apoio à Rede de Atenção Oncológica. Diretrizes brasileiras para o rastreamento do câncer do colo do útero / Instituto Nacional de Câncer. Coordenação Geral de Ações Estratégicas. Divisão de Apoio à Rede de Atenção Oncológica. - Rio de Janeiro: INCA, p. 104, 2011.

7. CESAR MAP, ANTUNES LB, AGUIAR RM. Existe a Constipação após Histerectomia? Avaliação Clínica e Manométrica. Revista brasileira de Coloproctologia, vol. 30 n. 2, 2010.

8. COSTA FKM, WEIGERT SP, BURCI L, et al., Os Desafios do Enfermeiro Perante a Prevenção do Câncer do Colo do Útero. Revista Gestão \& Saúde. Rio Grande do Sul, v. 17, n.1, p. 55-62, 2017.

9. CUNHA, ES. Assistência de Enfermagem na Prevenção do Câncer de Colo Uterino. FACIDER Revista Científica N9. 2015. Disponível em: http://sei-cesucol.edu.br/revista/index.php/facider/article/view/14. Acesso em 13 mar 2019

10. DUARTE MTC, AYRES JA, SIMONETTI JP. Consulta de enfermagem: estratégia de cuidado ao portador de hanseníase em atenção primária. Texto Contexto Enfermagem., v.18, n.1, p.100-7, 2009.

11. FERREIRA MH, SALES MC. Saúde Da Mulher Enquanto Políticas Públicas. Salus Journal of Health Sciences, v.3, n.2, p. 58-65, 2017.

12. FREITAS HG, SILVA MA, THULER LCS. Câncer do Colo do Útero no Estado de Mato Grosso do Sul: Detecção Precoce, Incidência e Mortalidade. Revista Brasileira de Cancerologia, v.58, n.3, p. 399-408, 2012.

13. FRIGATO S, Hoga LAK. Assistência à mulher com câncer de colo uterino: o papel da enfermagem. Revista Brasileira de Cancerologia, v.49, n.4, p. 209-214, 2003.

14. LEMOS A. Atenção Integral Á Saúde Da Mulher: O Olhar De Mulheres Que A Construíram. Revista de Enfermagem do Centro Oeste Mineiro, v.1, n.2, p. 220-227, 2011.

15. MELO MCSC, VILELA F, SALIMENA AMO, et.al., O Enfermeiro na Prevenção do Câncer do Colo do Útero: O Cotidiano da Atenção Primária. Revista Brasileira de Cancerologia., v. 58, n.3, p.389-98, 2012.

16. MISTURA C, SILVA RCCS, SALES JRP, et.al., Papel do Enfermeiro na Prevenção do Câncer de Colo Uterino na Estratégia Saúde da Família. Revista Contexto \& Saúde. v. 10, n. 20, 2011.

17. NAKAGAWA JTT, SCHIRMER J, BARBIERI M. Vírus HPV e câncer de colo de útero. Revista Brasileira de Enfermagem, v. 63, n. 2, 307-311 São Paulo, 2010. 
18. PEREIRA GG, SILVA JGF, BIZELLI DFP, SIMONATO LE. Fluorescência óptica no diagnóstico de lesões em colo cervical, Arch Health Invest, v.7, n.12, 2018.

19. PINTO JEP, AQUINO R, MEDINA MG, et.al., Efeito da Estratégia Saúde da Família nas internações por condições sensíveis à atenção primária em menores de um ano na Bahia, Brasil. Caderno de Saúde Pública, v. 34, n. 2, 2018.

20. SILVA DSM, SILVA AMN, BRITO LMO, at.al., Rastreamento do câncer do colo do útero no Estado do Maranhão, Brasil. Ciências de Saúde Coletiva, v. 19, n. 04, 2014.

21. SILVEIRA BL, MAIA RCB, CARVALHO MF. A. Câncer de Colo do Útero: papel do enfermeiro na Estratégia de Saúde da Família. Revista Científica da Faculdade de Educação e Meio Ambiente. Ariquemes: FAEMA, v. 9, n. 1, 2018.

22. WHO. Word Health Organization. Comprehensive cervical cancer control: a guide to essential practice (2nd ed.). Geneva, WHO Press, 2014.

23. WHO. Word Health Organization. International agency for research on cancer: globocan 2008. In: . World Cancer Report. Lyon: WHO, p. 11-104, 2008.

24. ZARDO GP, FARAH FP, MENDES FG, et.al., Vacina como agente de imunização contra o HPV. Ciências de Saúde Coletiva, v. 19, n. 9, 2014. 